\title{
Austerity policy and child health in European countries: a systematic literature review
}

\author{
Luis Rajmil ${ }^{1}$, Anders Hjern² ${ }^{*}$ (D) Nick Spencer ${ }^{3}$, David Taylor-Robinson ${ }^{4}$, Geir Gunnlaugsson ${ }^{5}$ and Hein Raat ${ }^{6}$
}

\begin{abstract}
Background: To analyse the impact of austerity measures taken by European governments as a response to the 2008 economic and financial crisis on social determinants on child health $(\mathrm{SDCH})$, and child health outcomes $(\mathrm{CHO})$.

Methods: A systematic literature review was carried out in Medline (Ovid), Embase, Web of Science, Psyclnfo, and Sociological abstracts in the last 5 years from European countries. Studies aimed at analysing the Great Recession, governments' responses to the crisis, and its impact on SDCH were included. A narrative synthesis of the results was carried out. The risk of bias was assessed using the STROBE and EPICURE tools.

Results: Fourteen studies were included, most of them with a low to intermediate risk of bias (average score 72.1\%). Government responses to the crisis varied, although there was general agreement that Greece, Spain, Ireland and the United Kingdom applied higher levels of austerity. High austerity periods, compared to pre-austerity periods were associated with increased material deprivation, child poverty rates, and low birth weight. Increasing child poverty subsequent to austerity measures was associated with deterioration of child health. High austerity was also related to poorer access and quality of services provided to disabled children. An annual reduction of $1 \%$ on public health expenditure was associated to $0.5 \%$ reduction on Measles-Mumps-Rubella vaccination coverage in Italy.

Conclusions: Countries that applied high level of austerity showed worse trends on SDCH and $\mathrm{CHO}$, demonstrating the importance that economic policy may have for equity in child health and development. European governments must act urgently and reverse these austerity policy measures that are detrimental to family benefits and child protection.
\end{abstract}

Keywords: Austerity, Child health, Child poverty, Economic crisis, Great recession, Social inequalities

\section{Background}

The Great Recession was characterised by an economic and financial crisis following the bail out of the banks in 2008. There followed a decline in gross domestic product (GDP) and high levels of unemployment, affecting European countries and worldwide [1]. Nevertheless, the magnitude of the crisis, its size, duration and geographical spread was different

\footnotetext{
* Correspondence: anders.hjern@su.se

${ }^{2}$ Centre for Health Equity Studies (CHESS), Stockholm University/Karolinska Institutet, SE-106 91 Stockholm, Sweden

Full list of author information is available at the end of the article
}

across European countries. Some countries experienced more substantial and sustained falls in GDP and rises in unemployment than others.

From 2010 to 11 onwards most European countries adopted austerity measures in response to the recession. The term austerity refers to measures taken by Governments to approach deficit reduction strategies following the financial crisis of 2008, primarily though limits to government expenditures and tax increases, and independently on the methods used to assess it. These austerity measures taken by governments, mainly characterised by reducing

(c) The Author(s). 2020 Open Access This article is licensed under a Creative Commons Attribution 4.0 International License, which permits use, sharing, adaptation, distribution and reproduction in any medium or format, as long as you give appropriate credit to the original author(s) and the source, provide a link to the Creative Commons licence, and indicate if changes were made. The images or other third party material in this article are included in the article's Creative Commons licence, unless indicated otherwise in a credit line to the material. If material is not included in the article's Creative Commons licence and your intended use is not permitted by statutory regulation or exceeds the permitted use, you will need to obtain permission directly from the copyright holder. To view a copy of this licence, visit http://creativecommons.org/licenses/by/4.0/. The Creative Commons Public Domain Dedication waiver (http://creativecommons.org/publicdomain/zero/1.0/) applies to the data made available in this article, unless otherwise stated in a credit line to the data. 
social spending and increasing taxation, were neither homogeneous nor similarly implemented; some protected public sector programs and systems while others instituted large budget cuts in education, health, and other public services [2].

The impact of the economic crisis on child health has been widely analysed. Studies suggest that the economic crisis disproportionately affected the most vulnerable groups, mainly children and youth from disadvantaged families [3]. Most studies of the impact of economic crisis on health have not distinguished between economic crises themselves and policy responses to these crises [4]. Although it would be difficult to disentangle the impact of the crisis itself from the Governments' responses [5], some studies have attempted to separately analyse this effect on the general population, mainly addressed specifically at adult health [6]. Less attention has been addressed to the impact of austerity measures on social determinants of child health $(\mathrm{SDCH})$ and child health outcomes $(\mathrm{CHO})[7,8]$.

Childhood is a vulnerable period to the main determinants of health. Factors related to financial, human and social capital have potential influence on future child health and development $[9,10]$. Moreover, there is evidence for the profound effects of social factors on health throughout childhood and into adulthood [11-13].

The objective of this systematic literature review was to analyse the impact of austerity measures taken by European governments as a response to the 2008 economic and financial crisis on the SDCH and $\mathrm{CHO}$. The hypothesis was that a higher level of austerity could be associated with a greater impact on inequalities and health, especially in the childhood population, where investment in the early lifecourse is essential to facilitate equitable and adequate child development, as well as the future adult health.

\section{Methods}

A systematic literature review was carried out in the databases Medline (Ovid), Embase, Web of Science, PsycInfo, and Sociological abstracts. Manual search for local published and unpublished documents were also included.

The Preferred Reporting Items of Systematic reviews Meta-Analyses (PRISMA, http://www.prisma-statement. $\mathrm{org} /$ ) guidelines was followed, although some items are not applicable given the characteristics of included studies. Search strategy adapted to each Database analysed included the terms "austerity", "financial crisis"," downturn", "child health outcomes", "child health inequalities", and "healthcare services" (search strategy available in the appendix).

\section{Inclusion criteria}

Included studies were those published in the last 5 years (from January 1rst, 2014 to November 4th, 2019) that reported on the impact of changes in family/children benefits, or in budgets, or access to healthcare system, or to social benefits as Government responses to the Great Recession on child health and living conditions (children $<18 y)$ in European countries. Studies should include the results of at least one European country, at least one $\mathrm{SDCH}$ or $\mathrm{CHO}$, separately from adult outcomes. Studies should analyse austerity and/or government responses to the crisis as the main exposure measure. Systematic or narrative reviews, longitudinal, before-after, qualitative and cross-sectional studies were included.

\section{Exclusion criteria}

Those studies reporting only the impact of the crisis were excluded, except when it could be linked to changes in policy responses; general population studies without stratification by age group or studies of adult populations, and Editorial or opinion papers were also excluded.

\section{Procedures}

Abstracts obtained by the initial search strategy were assessed for possible inclusion by two of the authors (AH, LR). Full text papers of the studies were obtained in doubtful cases and were independently assessed by these authors. Differences of opinion on inclusion were decided by discussion and consensus among all authors.

\section{Data extraction and analysis}

Data extracted included: setting (according to the country: international, national or regional study); type of study (trends of repeated cross-sectional data, beforeafter comparative study, cross-sectional study, review, etc); objective of study; years covered by the study; the specific target population (age range); measures of exposure, including austerity measures; outcome measures; and results in terms of impact on SDCH and/or $\mathrm{CHO}$.

The risk of bias of included studies was assessed by 4 of the authors (AH, DTR, NS, LR) using the Strengthening the Reporting of Observational Studies in Epidemiology (STROBE) initiative for quantitative studies [14]. Qualitative studies were assessed using EPICURE (Engagement, Processing, Interpretation, Critique Usefulness, Relevance and Ethics) [15]. STROBE items not applicable in certain study designs due to the inclusion of indicators based on ecological data (i.e., items related to characteristics of the individuals included in these indicators) were excluded from the evaluation of these specific studies. Thus, the average score between evaluators was calculated and subsequently the percentage achieved by each study on the total items evaluated. Risk of bias was stratified as high $(<50 \%)$, intermediate $(50-75 \%)$ and low $(>75 \%)$. For qualitative studies, 4 out of the 7 items (P, I, U, R) were considered essential to assess studies as average or low risk of bias (the 
latter if also met at least one of the other items). The lack of one or more of these 4 essential items was considered as high risk of bias. Analysis was stratified regarding the type of studies, the exposure measure of austerity, and the main outcome measures $(\mathrm{SDCH},[\mathrm{CHO}]$, and healthcare, and preventive services). A meta-analysis was not carried out given the nature of the study design and heterogeneity of the findings. A narrative synthesis of the results was carried out.

\section{Results}

Figure 1 shows the results of the literature search; 14 studies met the inclusion criteria.

\section{Characteristics of studies and exposure measures}

Table 1 spells out the characteristics of included studies: study design (cross-sectional, trends over time, beforeafter approach, cohort study, or qualitative study), country (individual country, or the number of countries analysed), exposure (type of austerity measure and source of data), outcome (SDCH and/or $\mathrm{CHO}$ ), and the risk of bias. Nine studies report time trend analyses of cross-sectional repeated data and two studies report before-after analyses following the implementation of austerity measures. The level of austerity measures was assessed by methods proposed by the International Monetary Fund (IMF) [21], the European Union's Maastricht criteria [16], or by analysing spending on families/children social protection in specific periods of time [17, 20].

A before-after approach was used to compare Italy, Greece, France and the UK [26], in terms of the ability of social benefits to reduce child poverty. One crosssectional study collected information on a survey to families of disabled children and professionals from 32 European countries [16], while a follow-up of a cohort of children from Ireland reported on the consequences of reported reductions on welfare benefits [28], and a qualitative study from Scotland provided information on

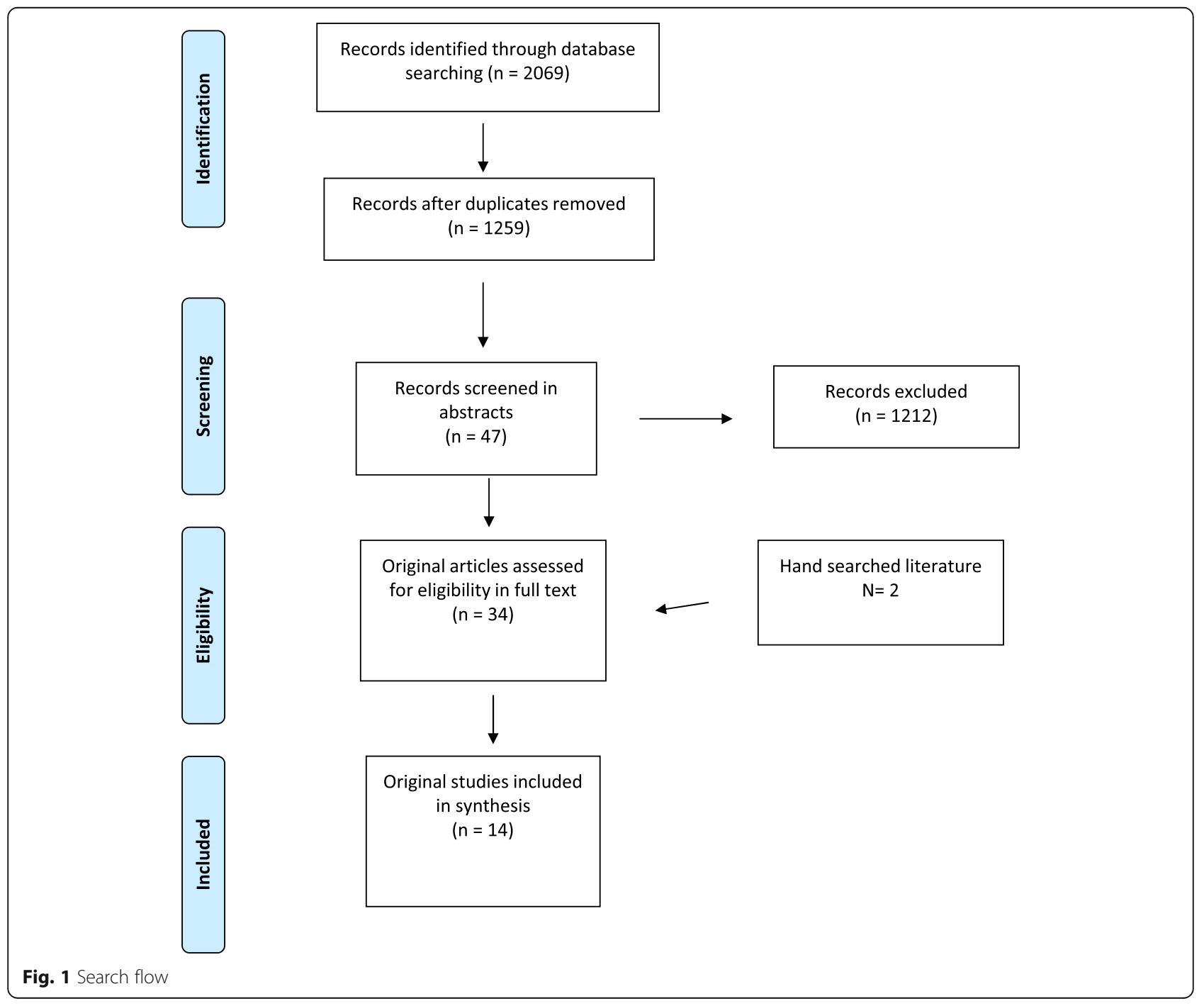


Table 1 Type of studies and risk of bias

\begin{tabular}{|c|c|c|c|c|}
\hline $\begin{array}{l}\text { Type of study } \\
\text { (first author, year) }\end{array}$ & Country/-ies & $\begin{array}{l}\text { Exposure measure } \\
\text { (year/s of study, source of data) }\end{array}$ & Outcome measure & $\begin{array}{l}\text { Risk of bias } \\
\text { (\% of the total } \\
\text { score-STROBE) }\end{array}$ \\
\hline
\end{tabular}

\section{Cross-sectional}

Horridge et al. 2019 [16] 32 EU countries

\section{Trends over time - Repeated} cross-sectional analysis

Chzhen et al. 2017 [17]

30 EU countries (27 EU plus Iceland, Norway, Switzerland)

Gunnlaugsson 2015 [18] Iceland

Herranz-Aguayo et al. Spain and Portugal

2016 [19]

Nygard et al. 2019 [20] 22 EU countries

Rajmil et al. 2018 [21] 16 EU countries

Rajmil et al. 2015 [22] Spain

Robinson et al. 2019 [23] England

Toffolutti et al. 2018 [24] Italy

Zografaki et al. 2018 [25] Greece

\section{Before-after approach}

D'Agostino et al.2019 [26] Italy, Greece, France, United Kingdom

Stefansson et al. 2018 [27] Iceland

\section{Cohort study}

Reinhard et al. 2018 [28] Ireland

\section{Qualitative study}

Stalker et al. 2015 [29] Scotland
Survey to professionals and families/ children. Countries classified according to the level of austerity following the European Union's Maastricht criteria (2016-17)

Spending on Social protection as a share of GDP (EU-SILC) (2008-13)

Governmental responses to the crisis (2004-14)

Government investment in family function (EU-SILC)

Public expenditure on family cash benefits and in-kind transfer benefits (OECD) (2006-15)

Countries stratified in 3 austerity groups according to the CAPB (IMF) (2005-15)

Government responses to the crisis (2005-13)

Effects during and after the English inequality strategy (1998-2010 / 2011-17)

Public health expenditure 2000-14

Changes in perinatal outcomes centered on the long term trends (1980-2004 and 2004-14)

Changes in social protection benefits (EU-SILC) (2009/14)

People own assessment of their ability to make ends meet (EU-SILC) (2009/14)

Cohort GUI; 3 waves included a question on reduction in social welfare benefits (2009/11/13)

Survey to providers for disabled children, and focus groups with carers and children (2011-13)
Healthcare services to disabled children. Requests on changes in the quality and characteristics of services in the last years

Relative and anchored (2008) child poverty rates

Low

Social determinants and child health

Low

Child poverty rates and AROPE Intermediate taxes

$(62.5)^{a}$

Child poverty rates (EU-SILC)

Low

$(87.5)^{a}$

Material deprivation, child

Low poverty, perinatal outcomes $\quad(82.5)^{\mathrm{a}}$ (EU-SILC), (OECD)

Social determinants, child health, and HCS

Intermediate $(67.5)^{a}$

IM according to the Towsend Low

index of deprivation area in $\quad(83.7)^{\mathrm{a}}$ quintiles

MMR coverage by health region Low

$(82.5)^{a}$

Perinatal outcomes at early Intermediate (2008-10) and "established crisis" (67) (2011-14)

Monetary and non-monetary indicators of well-being

Intermediate

Material deprivation by

$(75)^{a}$

dimensions, vulnerability

High

$(46.25)^{a}$

Family living conditions; child Low health, etc

Changes in access and quality STROBE: High (47.7) EPICURE (intermediate risk)

${ }^{a}$ Twenty was considered as the maximum score for STROBE given that a couple of items were not applicable. $A R O P E$ At risk of poverty and social exclusion, $C A P B$ Cyclically Adjusted Primary Balance, EU-SILC European Union Survey on Income and Living Conditions, GDP Gross Domestic Product, GUI Growing Up in Ireland, IMF International Monetary Fund, OECD Organisation for Economic Cooperation and Development, HCS Healthcare services, MMR Measles, mumps, rubella immunisation, STROBE STrengthening the Reporting of OBservational studies in Epidemiology (score $=0$ to 22) 
the impact of austerity measures on families with disabled children [29].

\section{Risk of bias}

Seven out of 14 studies showed a low risk of bias according to STROBE (average score $=72.1 \%$; median $=75.6 \%$ ), 2 studies showed high risk of bias, while the qualitative study showed intermediate risk of bias on the EPICURE assessment.

\section{Social determinants of child health (SDCH) (Table 2)}

The study analysing indicators of child poverty and material deprivation in 16 European countries according to the extent of austerity implemented by governments found that material deprivation increased in the period 2012-2015 in countries with high austerity level (interaction austerity"period 2012-15 = B: 5.62: $p<0.001$ ) [21]. Children were significantly less likely to be poor in countries with higher levels of social protection spending in 2008-2013 in $30 \mathrm{EU}$ countries [17]. Spending on in-kind benefits was more effective in reducing child poverty $(B=-1.6)$ than for cash benefits $(B=-1.2)$, although a gradual downward trend in the efficacy of both coefficients over time was reported [20]. Child and family poverty rates increased in Spain and
Portugal following the crisis partly due to low levels of social protection; social transfers in both countries only reduced poverty and social exclusion by $7.4 \%[19,22]$.

Shares of the social benefits devoted to family/children in the UK and France were approximately double those in Italy and Greece. In the study from D'Agostino et al., UK was one of the countries in which deterioration in some of the measures of well-being was avoided [26]. In Iceland, both children's deprivation and economic vulnerability were measured at higher levels in 2014 than in 2009 , though only the change in the latter was statistically significant [27]. Rates of deprivation and vulnerability were low and the overlap very limited, which may be indicative of low deprivation rates [18, 27].

\section{Child health outcomes (CHO)}

Results on $\mathrm{CHO}$ are summarised in Table 3. Increasing rates of low birth weight (LBW) were associated to high level of austerity in the study of 16 countries (interaction austerity" period 2012-15, B: $0.25 ; p=0.004$ ) [21]. Preterm birth and LBW increased by 37 and $7 \%$ respectively in Greece the years 2011-14 [25]. In Iceland, small for gestational age increased from 2 to $3.4 \%$ [18].

Table 2 Social determinants of child health (SDCH): child poverty, material deprivation, and social inequalities

\begin{tabular}{|c|c|}
\hline First author (year) & Main results \\
\hline Rajmil et al. 2018 [21] & $\begin{array}{l}\text { Material deprivation increased during the period } 2012-15 \text { in those countries with higher austerity } \\
\text { (interaction austerity*period } 2012-15=\text { B: } 5.62: p<0.001 \text { ) }\end{array}$ \\
\hline Chzhen et al. 2017 [17] & $\begin{array}{l}\text { Children were significantly less likely to be poor in countries with higher levels of social protection } \\
\text { spending in 2008-2013, even after controlling for the socio-demographic structure of the } \\
\text { population, per capita GDP and the working age unemployment rate. The effects of spending were } \\
\text { larger and more precisely estimated for relative rather than anchored poverty, and it was not } \\
\text { statistically significant in the last } 2 \text { years of the study }(2012 / 13)\end{array}$ \\
\hline
\end{tabular}

Nygard et al. 2019 [20]

D’Agostino et al. 2019 [26]

Herranz-Aguayo et al. 2016 [19]

Stefansson et al. 2018 [27]

Gunnlaugsson 2015 [18]
Child poverty: the coefficients for spending on in-kind benefits $(B=-1.6)$ were negative and consistently stronger than for cash benefits $(B=-1.2)$, even when controlling for other variables. There was also a gradual downward trend in the strengths of both coefficients as well as in the $\mathrm{R}$ Squares over time, which indicates that both forms of spending have become less efficient in reducing poverty over the studied period. This result can at least partly be attributed to higher unemployment of parents and a lower up-take rate of services (such as childcare services), as well as to cuts in the generosity of cash transfers to families

The shares of the social benefits devoted to the Family/Children function were approximately double in the UK and France than those of Italy and Greece. The higher and lower level of expenses in family/children benefits were for Italy: 5.4\% (2014) and 4.1\% in 2010; Greece: 4.4\% (2014) and 3.5\% in 2012; France: 8.6\% (2007) and 7.6\% in 2014; UK: 11.3\% (2010) and 10.3\% in 2013.

In 2013, Portugal exceeds the average on EU child poverty by almost 3\% and Spain by almost 7\% points. In Spain, one in three households is below the poverty thresholds (33.9\%), followed by Portugal (31.1\%). In Spain and Portugal, the ability to reduce poverty rates is much lower, remaining below the EU average (EU-15: 9.6\% and EU-27: 9.1\%), since both countries only achieved to reduce by $7.4 \%$ the risk of poverty and social exclusion after social transfers

Both children's deprivation and economic vulnerability were measured at higher levels in 2014 than in 2009, though only the change in the latter was statistically significant. Rates of deprivation in individual dimensions was low and the overlap very limited, which may be indicative of low deprivation rates

Governmental responses gave prominence to redistribution, through taxes and the social protection system. A set of measures represented protection of children were specifically improved (mental health, maternity care, immunisation, etc.). Percentage of children living in poverty almost not modified 
Table $\mathbf{3}$ Child health and healthcare services

\begin{tabular}{|c|c|}
\hline First author & Main results \\
\hline \multicolumn{2}{|c|}{ Perinatal indicators and child health } \\
\hline Rajmil 2018 et al. [21] & $\begin{array}{l}\text { LBW increased during the period } 2012-15 \text { in those countries with higher austerity (interaction austerity* }{ }^{*} \text { period } \\
2012-15, B: 0.25 ; p=0.004 \text { ) }\end{array}$ \\
\hline Zografaki et al. 2018 [25] & $\begin{array}{l}\text { LBW increased (Standardised rate ratio, SRR }=1.07[1.06-1.09] \text { ), as well as preterm births (SRR }=1.39,[1.37-1.42] \\
\text { during established crisis. Some differences found according to maternal origin and age }\end{array}$ \\
\hline Gunnlaugsson 2015 [18] & $\begin{array}{l}\text { Governmental responses gave prominence to redistribution, through taxes and the social protection system. } \\
\text { A set of measures protected children and were specifically improved (mental health, maternity care, } \\
\text { immunisation). A few indicators worsened (i.e. small for gestation age changed from } 2 \text { to } 3.4 \% \text {.) }\end{array}$ \\
\hline Robinson et al. 2018 [23] & $\begin{array}{l}\text { Absolute inequalities on IMR increased in 1990-1999 (annual change between the most deprived local } \\
\text { authorities and the rest of England =0.03) decreased during the welfare strategy period 2000-2010 (-0.11) and } \\
\text { increased in 2011-2017 (0.04). The analysis suggests that it is increases in public spending on healthcare and } \\
\text { welfare that are associated with decreases in inequalities in the IMR. }\end{array}$ \\
\hline Reinhard et al. 2018 [28] & $\begin{array}{l}48 \% \text { in } 2011 \text { and } 60 \% \text { in } 2013 \text { reported a reduction in welfare benefits. Besides the effect of the crisis itself, it } \\
\text { was associated with an increased risk of reporting asthma ( } \beta=0.014,95 \% \mathrm{Cl}: 0.004,0.023) \text { and atopy symptoms } \\
(\beta=0.014,95 \% \mathrm{Cl}: 0.001,0.027) \text {. }\end{array}$ \\
\hline Rajmil et al. 2015 [22] & $\begin{array}{l}\text { Great impact on health of vulnerable children related to cutting budgets on housing, access to HCS, preschool } \\
\text { investment. Increasing number of children living in poverty. No impact on child health at general population } \\
\text { level but to the most vulnerable groups }\end{array}$ \\
\hline \multicolumn{2}{|l|}{ Mental health, and disability } \\
\hline Horridge et al. 2019 [16] & $\begin{array}{l}\text { Health care professionals reported worsening quality of services than } 3 \text { years ago: increased waiting times, and } \\
\text { less time allocated to see each child compared to } 3 \text { years ago, and worse working conditions in the last year. } \\
\text { Nine in every ten families reported worsening quality of services for their disabled children compared to } \\
3 \text { years ago. Families from countries with austerity cuts reported more difficult access to welfare support and } \\
\text { benefits. }\end{array}$ \\
\hline Stalker et al. 2015 [29] & $\begin{array}{l}\text { Reduction or withdrawal of services in a wide range of provision-social work, education, voluntary } \\
\text { organisations, health and professions allied to medicine. Examples of services that were not provided or } \\
\text { shortened. Closure of day centres. Voluntary sector survey: A Shift from Preventative Work to Crisis Intervention. } \\
\text { Increase in unmet needs. Some families waited between one and } 3 \text { years for assessments or services on child } \\
\text { mental health for diagnose, equipment and/or home extensions. Difficulty meeting the needs of children on } \\
\text { the autistic spectrum was a recurring theme }\end{array}$ \\
\hline
\end{tabular}

Preventive services

Toffolutti et al. 2018 [24] PHE fell by 2\% in the whole country between 2010 and 2014. By regions, each 1\% annual reduction was associated to $0.5 \%(0.36-0.65)$ reduction on the coverage on MMR

IMR Infant mortality rates, HCS healthcare services, LBW low birth weight, PHE Public Health Expenditure

Absolute inequalities in infant mortality rates (IMR) in England increased in 1990-1999 (annual changes between the most deprived local authorities and the rest of England: 0.03), decreased during the welfare strategy period 2000-2010 (-0.11) and increased in 2011-2017 (0.04) after the end of this strategy [23]. The study Growing Up in Ireland (GUI) found that $48 \%$ of participating families in 2011 (2y old children) and 60\% in 2013 (4y olds) reported a reduction in welfare benefits, and it was associated with an increased risk of reporting asthma and atopy symptoms in the latter period [28].

\section{Healthcare services, and preventive services}

In the study of 32 European countries, families from countries with high austerity level reported more difficulties in access to healthcare services and benefits for children with disability; professionals reported worse quality of services provided and increasing waiting time for visits [16].
Stalker et al., [29] in a study of Scotland showed a reduction or withdrawal of services in a wide range of provision-social work, education, voluntary organisations, health and professions allied to medicine. Their survey described a shift in the voluntary preventative work to crisis intervention and an increase in unmet needs. Waiting time for some families for assessments on child mental health services for diagnosis, equipment and/or home extensions were between one and 3 years. A recurring theme was the difficulty meeting the needs of children on the autistic spectrum [29].

An annual reduction of $1 \%$ in public health expenditure (PHE) was associated to $0.5 \%$ reduction in Measles-MumpsRubella (MMR) vaccination coverage by region in Italy [24].

\section{Discussion}

This systematic review of studies evaluating the consequences of austerity measures on child health and wellbeing results support the hypothesis that those countries that have applied and maintained high levels of austerity 
have experienced adverse consequences for children. These consequences include deteriorating social determinants as well as child health outcomes and reduced access to, and quality of, preventive and curative healthcare services. This negative impact on child health and development may have implications for the future adult health of a generation.

This review corroborates other studies at the European general population level $[1,6]$. Despite considerable crosscountry differences, these studies suggest that the interaction of fiscal austerity with economic shocks and weak social protection ultimately may lead to social crisis with a negative impact on health. In countries that applied high levels of austerity, another review at the European general population level showed an increase in homelessness, food insecurity, and worsening mental health and increase of suicide rates, as well as difficulties with access to care [2]. In Greece, Spain, UK, and France charities also reported marked rises in people seeking emergency food support coinciding with the introduction of austerity measures.

The present review shows significant variability in the situation of European countries before and during the crisis. Classification of countries according to the level of austerity also shows an important variability. However, the results are consistent with respect to the impact of austerity measures on $\mathrm{SDCH}$ and $\mathrm{CHO}$.

Poverty reduction strategies, either in-kind or cash benefits, were suggested to be less effective as a result of austerity measures employed by governments [17, 20]. Investment in family and child policies declined during the study period even in countries that traditionally invested more, and was clearly insufficient in countries such as Spain and Portugal $[19,26]$. And these changes coincide again, with the period of greatest adjustment for austerity.

The impact on children's health has been detected especially in perinatal indicators and in countries that have significantly reduced the healthcare budget, such as Greece [16]. The impact has been greater in families with vulnerable children and a very important change has been detected in the access to, and provision of, services for children with disabilities and in child mental health services. Child and adolescent mental health services in Greece operate with $10-40 \%$ fewer employees, and this situation coincides with a rise in demand for child psychiatric services but also to a qualitative change in the severity of psychopathology dealt with in everyday clinical practice [30].

Austerity measures have been reported to impact deprived groups the most [2]. In this sense, children represent a particularly vulnerable population group. Inequalities in early child development have been identified as a major contributing factor to inequalities in adult health [31]. Besides the studies included in the present review, the recent increase in infant mortality in the poorest areas of England associated with rising child poverty would also be associated to the impact of austerity policies [32], and the need to urgently address these policies for the real protection of families and children.

Although all but three of the included studies showed low risk of bias there are some limitations. First, the analysis of ecological data prevents the establishment of causal associations. Moreover, some studies comparing $\mathrm{SDCH}$ included ecologic exposure or outcome measures which prevents application of some items in STROBE. Nevertheless, almost all included studies show that a high level of austerity is associated with worse outcomes in SDCH. Secondly, differences in classifications used to assess the level of austerity make it difficult to establish comparisons and summary measures. Moreover, these classifications do not discriminate well between specific aspects of budget reduction, such as family benefits, early child investment, prenatal care, etc. It may not adequately reflect specific national policies that establish general economic rigor while trying to protect the most vulnerable groups, such as children. This could partly explain why Iceland [18], despite having a high level of austerity at the beginning of the economic crisis and in response to the crisis, showed trends more consistent with countries with low levels of austerity and protecting social benefits for children. This fact could be associated to a robust primary healthcare system with universal access and extensive preventive child health services, and governmental emphasis to protect low-income groups through redistribution of tax revenues and innovative labour market initiatives [33]. On the other hand, almost all included studies agree that countries such a Greece, Spain, Portugal or the UK applied higher levels of austerity than the rest. Thirdly, it was not possible to include a specific analysis of child maltreatment and trends in proportion of children in care and social protection due to the lack of reliable and comparable data at European level, and this should be the matter for future studies. Finally, there is a lack of studies of trends in gradients in social inequalities in child health, while studies at the general population level showed an increase in inequalities during the recession and austerity periods [34]. A study that analysed the investment per child in England according to the area of residence found a greater reduction in areas with greater deprivation [35]. In summary, this review highlights the need for more robust studies of the impact of austerity on child health in individual countries.

\section{Conclusions}

Countries that applied high levels of austerity showed worse trends on $\mathrm{SDCH}, \mathrm{CHO}$, and access to, and quality of, preventive and curative healthcare services, demonstrating the importance of economic policy for equity in child health and development [36]. European governments must act urgently and reverse these austerity policy measures that are detrimental to family benefits and child protection. 


\section{Supplementary information}

Supplementary information accompanies this paper at https://doi.org/10. 1186/s12889-020-08732-3.

\section{Additional file 1.}

\section{Abbreviations}

CHO: Child health outcomes; CAPB: Cyclically Adjusted Primary Balance; EPICURE: Engagement, Processing, Interpretation, Critique Usefulness, Relevance and Ethics; GDP: Gross Domestic Product; IMF: International Monetary Fund; SDCH: Social determinants of child health; STROBE: Strengthening the Reporting of Observational Studies in Epidemiology

\section{Acknowledgments}

Authors would like to thank Magdalena Svanberg \& GunBrit Knutssön, Librarians from the Karolinska Institutet for their help in the bibliographic search.

\section{Authors' contributions}

$L R$, NS and $A H$ were involved in the initial conception and design of the study. LR and AH extracted data and NS, DTR, GG and HR participated in the data analysis strategy. All authors were involved in the interpretation and discussion of results through call conferences. LR developed the first draft of the manuscript. All authors have read and approved the manuscript. LR is study guarantor.

\section{Funding}

No funding was received for this article. Open access funding provided by Stockholm University.

\section{Availability of data and materials}

The datasets used and/or analysed during the current study are available from the corresponding author on reasonable request.

\section{Ethics approval and consent to participate}

This study does not include individual data.

\section{Consent for publication}

Not applicable.

\section{Competing interests}

The authors declare that they have no competing interest.

\section{Author details}

${ }^{1}$ Retired, paediatrician and public health and epidemiology specialist, Homer 22 1rst 1, Barcelona, Spain. ${ }^{2}$ Centre for Health Equity Studies (CHESS), Stockholm University/Karolinska Institutet, SE-106 91 Stockholm, Sweden. ${ }^{3}$ Emeritus Professor of Child Health, Division of Mental Health and Wellbeing, Warwick Medical School University of Warwick, Coventry CV4 9JD, UK. ${ }^{4}$ Clinical Senior Lecturer, Department of Public Health and Policy, Whelan Building University of Liverpool, Liverpool, UK. ${ }^{5}$ Faculty of Sociology, Anthropology and Folkloristics, University of Iceland, Reykjavik, Iceland. ${ }^{6}$ Department of Public Health, Erasmus Medical Center, University Medical Center, PO Box 2040, 3000, CA, Rotterdam, The Netherlands.

Received: 27 February 2020 Accepted: 19 April 2020

Published online: 19 May 2020

\section{References}

1. Karanikolos M, Mladovsky P, Cylus J, et al. Financial crisis, austerity, and health in Europe. Lancet. 2013;381(9874):1323-31. https://doi.org/10.1016/ S0140-6736(13)60102-6.

2. Stuckler D, Reeves A, Loopstra R, Karanikolos M, McKee M. Austerity and health: the impact in the UK and Europe. Eur J Pub Health. 2017. 27(November):18-21. https://doi.org/10.1093/eurpub/ckx167.

3. Rajmil L, de Sanmamed M-JF, Choonara I, et al. Impact of the 2008 economic and financial crisis on child health: A systematic review. Int J Environ Res Public Health. 2014:11(6). https://doi.org/10.3390/ ijerph110606528.
4. Barr B, Taylor-Robinson D. Recessions are harmful to health. BMJ. 2016; 354(September):1-2. https://doi.org/10.1136/bmj.i4631.

5. Dahl E, Saltkjel T, Ingelsrud MH, Halvorsen K. A fuzzy set approach to economic crisis, austerity and public health. Part I. European countries' conformity to ideal types during the economic downturn. Scand J Public Health. 2017:45(18_suppl):41-7. https://doi.org/10.1177/1403494817706632.

6. Saltkjel T, Ingelsrud MH, Dahl E, Halvorsen K. A fuzzy set approach to economic crisis, austerity and public health. Part II: How are configurations of crisis and austerity related to changes in population health across Europe? Scand J Public Health. 2017:45(18_suppl):48-55. https://doi.org/10. $1177 / 1403494817707125$

7. Torjesen I. Austerity cuts are eroding benefits of sure start children's centres. BMJ. 2016;532:i335. https://doi.org/10.1136/bmj.i335.

8. Taylor-Robinson D, Wickham S, Barr B. Child health at risk from welfare cuts: poverty has an enduring influence on children's development, health outcomes, and survival. BMJ. 2015;351. https://doi.org/10.1136/bmj.h5330.

9. Dalhlgren G, Whitehead M. Policies and Strategies to promote social equity in health. Background Document to WHO - Strategy Paper for Europe.; 1991. doi: DOl::

10. WHO/UNICEF/WB. Nurturing Care for Early Childhood Development: A Framework for helping children survive and thrive to transform health and human potentia, vol. 37; 2018. https://apps.who.int/iris/bitstream/handle/1 0665/272603/9789241514064-eng.pdf.

11. Galobardes B, Lynch JW, Davey SG. Is the association between childhood socioeconomic circumstances and cause-specific mortality established? Update of a systematic review. J Epidemiol Community Health. 2008;62(5): 387-90. https://doi.org/10.1136/jech.2007.065508.

12. Hirsch D, Spencer N. Unhealthy lives; intergenerational links between child poverty and poor health in the UK, summary briefing. 2008;(1099008):8-11.

13. Al N, Román JC, Gross CL, Gilman SE, Loucks EBBS. Early childhood social disadvantage is associated with poor health behaviours in adulthood. Ann Hum Biol. 2016:43(2):144-53.

14. von Elm E, Altman DG, Egger M, Pocock SJ, Gøtzsche PC, Vandenbroucke JP. The strengthening the reporting of observational studies in epidemiology (STROBE) statement: guidelines for reporting observational studies. Int J Surg. 2014:12(12):1495-9. https://doi.org/10.1016/j.ijsu.2014.07.013.

15. Stige $B$, Malterud $K$, Midtgarden T. Toward an agenda for evaluation of qualitative research. Qual Health Res. 2009;19(10):1504-16. https:/doi.org/ 10.1177/1049732309348501.

16. Horridge KA, Dew $\mathrm{R}$, Chatelin A, et al. Austerity and families with disabled children: a European survey. Dev Med Child Neurol. 2019;61(3):329-36. https://doi.org/10.1111/dmcn.13978.

17. Chzhen Y. Unemployment, social protection spending and child poverty in the European Union during the great recession. J Eur Soc Policy. 2017;27(2): 123-37. https://doi.org/10.1177/0958928716676549.

18. Gunnlaugsson G. Child health in Iceland before and after the economic collapse in 2008. Arch Dis Child. 2016;101(5):489-96. https://doi.org/10.1136/ archdischild-2014-307196.

19. Herranz Aguayo I, Herraiz ED, Marques EM, Machado I, Almeida S. Child at risk of poverty or social exclusion: Comparative view between Spain and Portugal in the European context. Soc Indic Res. 2016;129(3):961-78. https:// doi.org/10.1007/s11205-015-1119-y.

20. Nygård $M$, Lindberg $M$, Nyqvist $F$, Härtull $C$. The role of cash benefit and inkind benefit spending for child poverty in times of austerity: an analysis of 22 European countries 2006-2015. Soc Indic Res. 2019;146(3):533-52. https://doi.org/10.1007/s11205-019-02126-8.

21. Rajmil L, Taylor-Robinson D, Gunnlaugsson G, Hjern A, Spencer N. Trends in social determinants of child health and perinatal outcomes in European countries 2005-2015 by level of austerity imposed by governments: A repeat cross-sectional analysis of routinely available data. BMJ Open. 2018; 8(10). https://doi.org/10.1136/bmjopen-2018-022932.

22. Rajmil L, Siddiqi A, Taylor-Robinson D, Spencer N. Understanding the impact of the economic crisis on child health: The case of Spain. Int J Equity Health. 2015;14(1). https://doi.org/10.1186/s12939-015-0236-1.

23. Robinson T, Brown H, Norman PD, Fraser LK, Barr B, Bambra C. The impac of New Labour's English health inequalities strategy on geographical inequalities in infant mortality: a time-trend analysis. J Epidemiol Community Heal. 2019;73(6):564-8. https://doi.org/10.1136/jech-2018211679.

24. Toffolutti V, McKee M, Melegaro A, Ricciardi W, Stuckler D. Austerity, measles and mandatory vaccination: cross-regional analysis of vaccination in Italy 
2000-14. Eur J Pub Health. 2019;29(1):123-7. https://doi.org/10.1093/eurpub/ cky178.

25. Zografaki I, Papamichail D, Panagiotopoulos T. Adverse effect of the financial crisis in Greece on perinatal factors. Eur J Public Health. 2018;28(6): 1116-21. https://doi.org/10.1093/eurpub/cky078.

26. D'Agostino A, Gagliardi F, Giusti C, Potsi A. Investigating the impact of the economic crisis on children's wellbeing in four European countries. Soc Sci Res. 2019;84:102322. https://doi.org/10.1016/j.ssresearch.2019.06.013.

27. Stefansson KH, Arnardottir L, Karlsson AO. Children's deprivation and economic vulnerability in Iceland 2009 and 2014. Child Indic Res. 2018;11(3): 783-803. https://doi.org/10.1007/s12187-017-9492-5.

28. Reinhard E, Layte R, McCrory C, Panico L, Avendano M. The Great Recession and the health of young children: A fixed-effects analysis in Ireland. Am J Epidemiol. 2018;187(7):1438-48. https://doi.org/10.1093/aje/kwy001.

29. Stalker K, MacDonald C, King C, McFaul F, Young C, Hawthorn M. "We could kid on that this is going to benefit the kids but no, this is about funding": Cutbacks in services to disabled children and young people in Scotland. Child Care Pract. 2015;21(1):6-21. https://doi.org/10.1080/13575279.2014. 972341.

30. Anagnostopoulos DC, Giannakopoulos G, Christodoulou NG. The synergy of the refugee crisis and the financial crisis in Greece: Impact on mental health. Int J Soc Psychiatry. 2017;63(4):352-8. https://doi.org/10.1177/ 0020764017700444.

31. Pillas D, Marmot M, Naicker K, Goldblatt P, Morrison J, Pikhart H. Social inequalities in early childhood health and development: a European-wide systematic review. Pediatr Res. 2014;76(5):418-24. https://doi.org/10.1038/pr. 2014.122.

32. Taylor-Robinson D, Lai ETC, Wickham S, et al. Assessing the impact of rising child poverty on the unprecedented rise in infant mortality in England, 2000-2017: time trend analysis. BMJ Open. 2019;9(10):1-6. https://doi.org/10. 1136/bmjopen-2019-029424.

33. Gunnlaugsson G, Einarsdóttir J. 'All's well in Iceland?' Austerity measures, labour market initiatives, and health and well-being of children. Nord välfärdsforskning | Nord Welf Res. 2016;1(01):30-42. https://doi.org/10.18261/ issn.2464-4161-2016-01-04.

34. Dahl E, Arntzen A, Giæver $\varnothing$. Welfare state and health inequalities under economic recession: Introduction. Scand J Public Health. 2017;45(18_suppl): 3-8. https://doi.org/10.1177/1403494817714916.

35. Webb CJR, Bywaters P. Austerity, rationing and inequity: trends in children's and young peoples' services expenditure in England between 2010 and 2015. Local Gov Stud. 2018;44(3):391-415. https://doi.org/10.1080/03003930. 2018.1430028 .

36. Spencer N, Raman S, O'Hare B, Tamburlini G. Addressing inequities in child health and development: towards social justice. BMJ Paediatr Open. 2019; 3(1):e000503. https://doi.org/10.1136/bmjpo-2019-000503.

\section{Publisher's Note}

Springer Nature remains neutral with regard to jurisdictional claims in published maps and institutional affiliations.

Ready to submit your research? Choose BMC and benefit from:

- fast, convenient online submission

- thorough peer review by experienced researchers in your field

- rapid publication on acceptance

- support for research data, including large and complex data types

- gold Open Access which fosters wider collaboration and increased citations

- maximum visibility for your research: over $100 \mathrm{M}$ website views per year

At BMC, research is always in progress.

Learn more biomedcentral.com/submissions 